\title{
Investigation of CVD graphene topography and surface electrical properties
}

\author{
Rui Wang ${ }^{1,2}$, Ruth Pearce ${ }^{1}$, John Gallop ${ }^{1}$, Trupti Patel ${ }^{1,3}$, Fang Zhao ${ }^{3}$, Andrew Pollard ${ }^{1}$, Norbert \\ Klein $^{2}$, Richard Jackman ${ }^{3}$, Amaia Zurutuza ${ }^{4}$ and Ling Hao ${ }^{1,2 *}$ \\ ${ }^{I}$ National Physical Laboratory, Teddington, TW11 OLW, UK \\ ${ }^{2}$ Department of Materials, Imperial College London, SW7 2AZ, UK \\ ${ }^{3}$ London Centre for Nanotechnology, University College London, WC1H OAH, UK \\ ${ }^{4}$ Graphenea S.A.,Tolosa Hiribidea, 76 E-20018 Donostia, San Sebastian, Spain
}

*Corresponding author: ling.hao@npl.co.uk

\begin{abstract}
Combining scanning probe microscopy techniques to characterise samples of graphene, a self-supporting single atomic layer hexagonal lattice of carbon atoms, provides far more information than a single technique can. Here we focus on graphene grown by chemical vapour deposition (CVD), grown by passing carbon containing gas over heated copper, which catalyses single atomic layer growth of graphene on its surface. To be useful for applications the graphene must be transferred onto other substrates. Following transfer it is important to characterise the CVD graphene. We combine atomic force microscopy (AFM) and scanning Kelvin probe microscopy (SKPM) to reveal several properties of the transferred film. AFM alone provides topographic information, showing 'wrinkles' where the transfer provided incomplete substrate attachment. Combined with SKPM which measures the surface potential (SP), indicating regions with different graphene layer numbers, local defects and impurities can also be observed in the SP scan. Finally, Raman spectroscopy can confirm the structural properties of the graphene films, such as the number of layers and level of disorder, by observing the peaks present. We report example data on a number of CVD samples from different sources.
\end{abstract}

\section{Key words}

\section{CVD Graphene, Raman, Scanning Kelvin probe microscopy}

\section{Introduction}

The CVD method for graphene growth is arguably the most promising method for high quality large area production [1]. However, each new set of growth parameters and transfer techniques requires characterisation to understand the effect the processes have on the quality of the graphene [2,3]. We explain the characterisation techniques used and why the information they provide is useful. We use these techniques to investigate high quality commercially available graphene films. We show that a film transferred onto quartz is predominantly mono-layer with some bi-layer regions, is very clean and relatively defect free. The quartz substrate affects the electronic properties of the film uniformly. We also investigate a CVD film which has been transferred onto a diamond substrate; the effect of the substrate on the electronic properties of the graphene is mapped using SKPM and the rough surface topography is shown to affect the electronic properties of the film differently due to 
the graphene being suspended over deep pits in the rough substrate. This graphene is also predominantly single-layer with SKPM showing only a few areas of bilayer graphene.

\section{Experimental Methods}

\subsection{Scanning Kelvin probe microscopy of graphene samples}

Scanning Kelvin probe microscopy (SKPM) was carried out in an NT-MDT Aura system or in a Bruker ICON system with $\mathrm{Si}$ on $\mathrm{SiN}$ cantilevers at room temperature in atmospheric conditions. On both systems a single-pass, frequency-modulated Kelvin probe microscopy mode was used. Topography is mapped by measuring the short range van der Waals forces giving the height of the sample. SKPM measures the longer range electrostatic forces acting on the cantilever, mapping the surface potential (SP) of the sample which is equivalent to the contact potential difference $\left(\mathrm{V}_{\mathrm{CPD}}\right)$ measured in a standard Kelvin probe experiment. The topography is mapped by resonating a cantilever near its resonant frequency and measuring the frequency shift due to forces acting on the cantilever near the sample surface. The SP is a measure of the changing work function of the sample and is calculated by applying a much lower frequency ac bias with a backing dc bias to the cantilever (not the piezo stack) than the resonance of the cantilever. The effect of the low frequency bias on the resonance of the cantilever is nullified by sweeping the backing dc bias, the dc bias at which the electrostatic forces are nullified is equivalent to the $\mathrm{V}_{\mathrm{CPD}}$ between the sample and the cantilever. If the work function of the tip is known the work function of the sample can be estimated from the equation $\Phi$ sample $=$ SP- $\Phi$ tip [4]. However, the precise work function may also depend on experimental parameters, tip condition, as well as doping due to substrate morphology [4] or the environment [5] whereas the difference in SP between two areas of the sample with different work function is not affected by tip condition but can be affected by environmental or substrate doping [6].

\subsection{Raman Spectroscopy of graphene layers}

Raman spectroscopy is an analytical technique which relies on the inelastic scattering of monochromatic light, typically from a laser source incident on the sample, for the identification of distinctive vibration modes of materials. Raman spectroscopy is recognised as an ideal characterisation tool for determining the structural, chemical and electronic properties of graphene due to the strong signal of the distinctive bands observed, and due to the rapid and non-destructive nature of this technique [7].

The Raman spectroscopy measurements were performed in ambient conditions using a LabRAM HR Evolution Raman system (Horiba Scientific, UK) with a $532 \mathrm{~nm}(2.33 \mathrm{eV})$ excitation laser and $100 \times, 0.9 \mathrm{NA}$ objective. In order to prevent sample damage from laserinduced heating of the surface measurements were performed with an incident laser power of $<1 \mathrm{~mW}$ for a laser spot size of $\sim 1 \mu \mathrm{m}$ diameter, and no significant changes in the Raman spectrum were observed over time for these parameters.

For graphene layers, the Raman G-peak at $\sim 1580 \mathrm{~cm}^{-1}$ occurs due to in-plane vibrations of sub lattice A against sub lattice B [8]. The dispersive D-peak at $\sim 1350 \mathrm{~cm}^{-1}$ is due to the breathing modes of six-atom rings, but requires a defect for its activation. The 2D-band at $\sim 2700 \mathrm{~cm}^{-1}$ is the D-peak overtone, but is observed without requiring a defect to be present $[9,10]$. 
The number of graphene layers can be determined from the Raman 2D-peak (also referred to as the G' peak), specifically the peak-shape for Bernal stacked graphene, or by measuring both the full width at half-maximum (FWHM) of the 2D-peak and the ratio of the $2 \mathrm{D} / \mathrm{G}$ peaks for turbostratic graphene $[1,12,13]$.

\section{Graphene characterisation results}

\subsection{CVD Graphene on quartz}

Single-layer CVD graphene was produced by Graphenea and transferred onto a quartz substrate. The topography (Fig. 1) shows that the graphene follows the polishing striations of the substrate and forms some wrinkles. The sample surface is very clean with little surface contamination in the form of dust or residual polymer from the transfer process. The SKPM map shows areas of brighter contrast which correspond to bi-layer patches and higher SP also on the wrinkles which are attributed to graphene formation on the grain boundaries of copper, which may be due to folding over of the graphene in these areas, or strips of bi-layer formation at the copper grain boundaries.
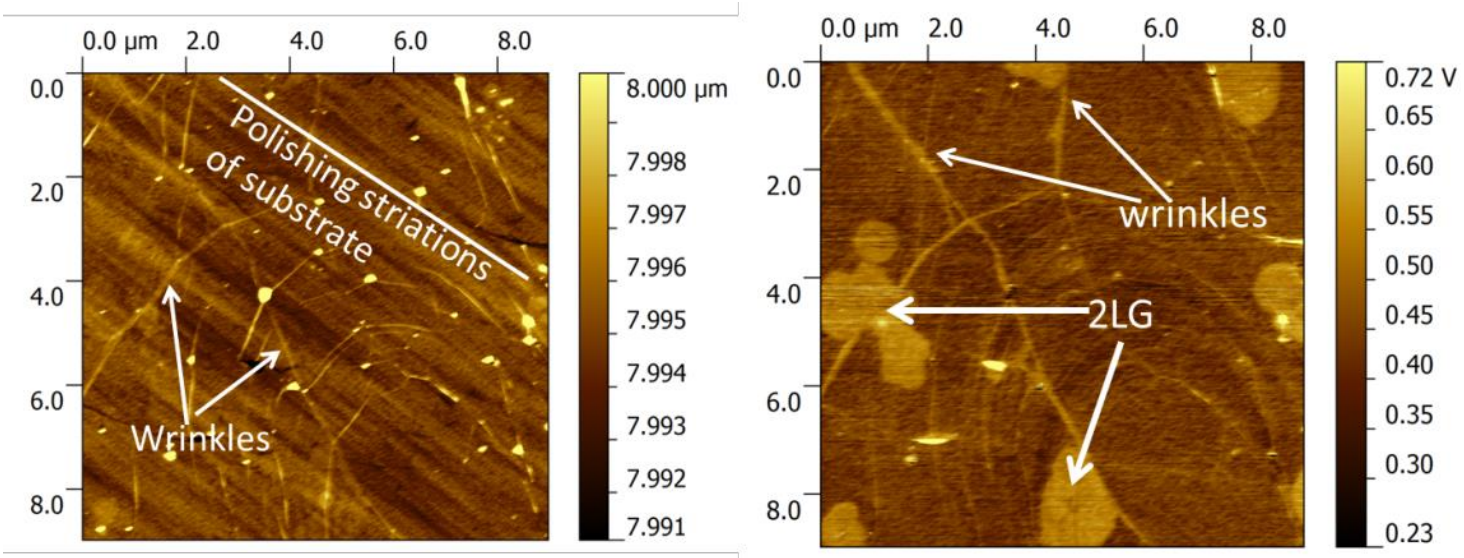

Figure 1: a) topography of CVD grown nominally single layer graphene transferred onto a quartz substrate showing wrinkles in the graphene and polishing marks on the underlying quartz substrate that are followed by the graphene b) the SKPM map of the same area showing higher SP for bi-layer areas and wrinkles in the graphene.

Fig. 2 shows the topography and SKPM image of the graphene sheet; the wrinkle formation on the graphene (Fig. 2a) resembles closely that of the domains of copper [14] suggesting that these wrinkles may follow the grain boundaries of the copper. The wrinkles have a similar change in SP as the bilayer graphene patches (Fig. c) and d)), in the wrinkled areas the graphene may be folded or the wrinkles may be bi-layer strips formed at the grain boundaries of the copper. The similarity between the SP difference between the mono-layer and the bilayer and the SP difference over a wrinkle suggests that there is bi-layer graphene at the wrinkles. The SP map shows that the 2LG areas are usually in close proximity to the grain boundaries. It is likely that the $2 \mathrm{LG}$ areas predominately nucleate at the wrinkles suggesting that these areas could be nucleation sites for growth. 

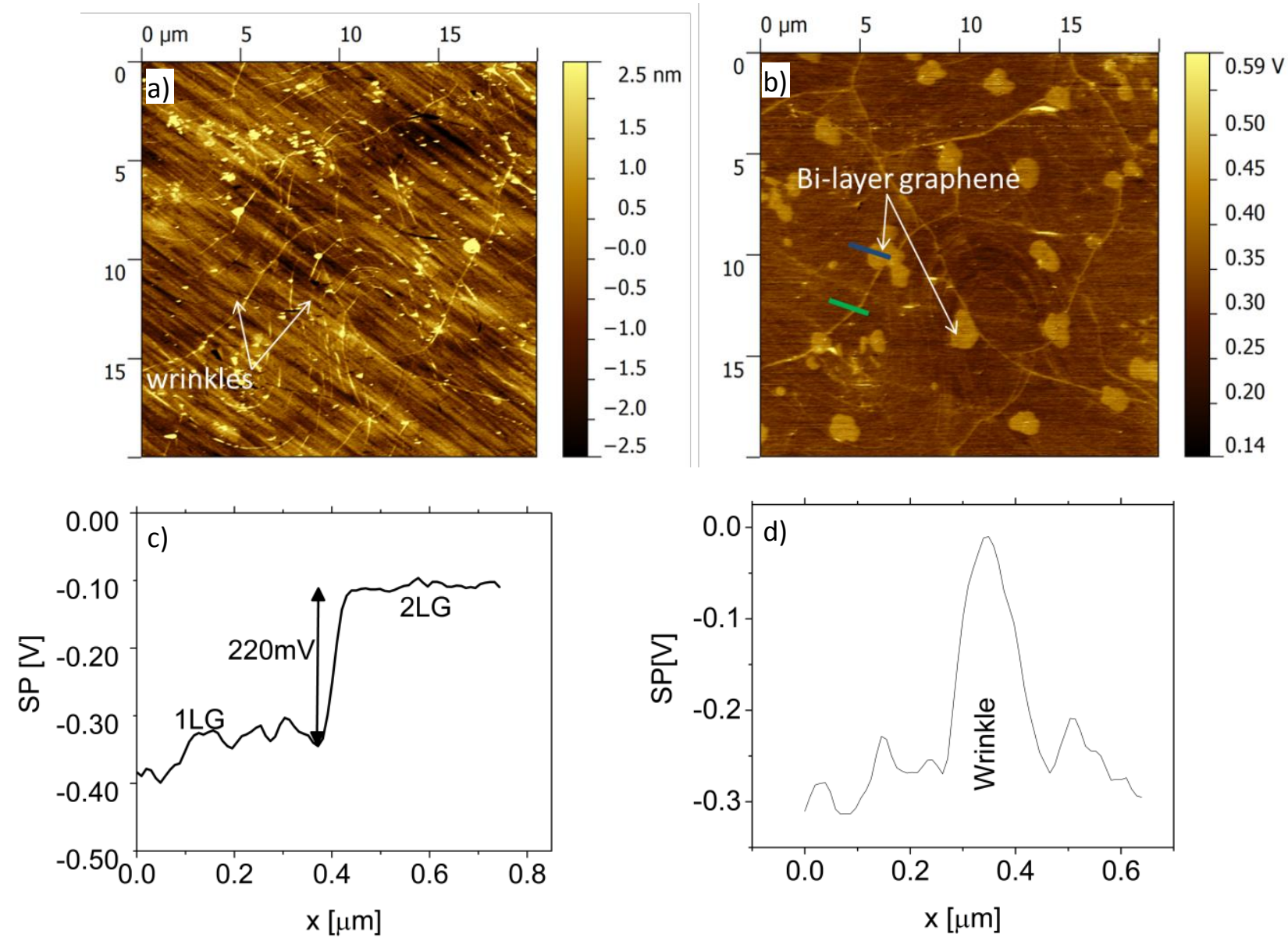

Figure 2: a) topography b) SKPM c) difference in SP between 1 and 2LG taken at the area marked in blue on figure b) d) SP change over a wrinkle taken from area marked in green on figure b).

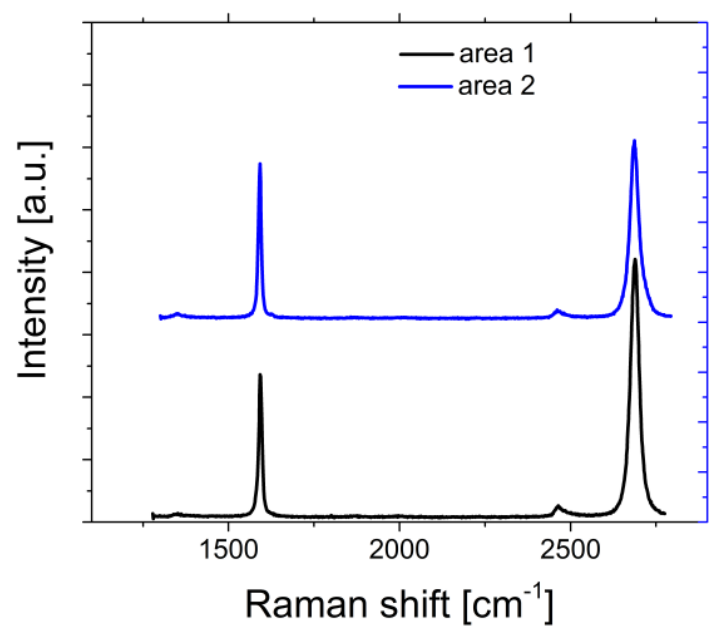

Figure 3: Raman spectra for the graphene sample from Graphenea showing the G- and 2D-peak, the 2D/G ratios reveal predominantly mono-layer in area 1 and bi-layer in area 2

The Raman spectrum of this sample consists of distinct peaks with the G-and 2D-peak clearly visible at $\sim 1580 \mathrm{~cm}^{-1}$ and $\sim 2700 \mathrm{~cm}^{-1}$ respectively (Fig. 3). The Raman spectra show monoand bi-layer graphene on the quartz substrate; the 2D-peak is fitted to one Lorentzian suggesting mono-layer or turbostratic multi-layers. The layer thickness is calculated to be mono-layer (area 1) with some bi-layer areas (area 2) from the 2D/G Raman intensity ratio 
$\left(\mathrm{I}_{2 \mathrm{D}} / \mathrm{I}_{\mathrm{G}}\right)$ and $2 \mathrm{D}$-peak FWHM. The $\mathrm{I}_{2 \mathrm{D}} / \mathrm{I}_{\mathrm{G}}$ ratio of the mono-layer area indicates some bi-layer contribution to the mono-layer regions $\left(\mathrm{I}_{2 \mathrm{D}} / \mathrm{I}_{\mathrm{G}}\right.$ ratio of $\sim 1.8$ rather than the expected $\mathrm{I}_{2 \mathrm{D}} / \mathrm{I}_{\mathrm{G}}>2$ for mono-layer CVD graphene, with an associated 2D-peak FWHM of $\sim 29 \mathrm{~cm}^{-1}$ [13] which is likely to be due to contributions from the folded or bi-layer graphene formed at the grain boundaries due to the Raman probe size.

\subsection{CVD graphene transferred onto diamond}

Through CVD methods, single crystal diamond substrates (SCD) with orientation of (100) and (111) were grown and used (Element Six, Ltd.). Prior to use, substrates were cleaned in strongly oxidising solutions to remove any residual organic contaminants and/or adsorbatehydrogenated surface complexes contributing to surface conductivity $[15,16]$. To clean the diamond the etch solution consisted of sulphuric acid and ammonium persulphate and the rinse solution was a mixture of hydrogen peroxide and ammonium hydroxide. After cleaning, all the samples were oxygen terminated. To produce hydrogenated surfaces, SCD samples were exposed to hydrogen microwave-stimulated plasma $\left(20 \mathrm{mbar}, 800^{\circ} \mathrm{C}, 10 \mathrm{~min}\right.$, Seki Technotron AX5010). The large area graphene on $\mathrm{Cu}$ foil was coated with PMMA, and subsequently the copper was etched away in a $\mathrm{FeCl}_{3}$ solution. Graphene was then transferred onto substrates, with acetone being used to remove the PMMA [17].

The topography image (Fig. 4) shows the roughness of the diamond with many deep pits observed on the surface. The graphene is expected to follow the diamond topography however the graphene may be suspended over some deep pits. The SKPM map appears inhomogeneous, however when comparing to the topography map there is correlation between the areas in the SKPM map which show darker areas corresponding to some of the deepest pits in the topography image. Here the graphene may be either suspended or the rough surface of the diamond may have created holes in the graphene. The brighter areas on the SP map are areas with a higher SP or a smaller work function. This may be due to the graphene being in close proximity to, or stretched over the diamond substrate, electron donation from areas of the diamond which have been modified by the hydrogen process or due to thicker regions of graphene. The roughness of the diamond surface makes it challenging to measure the thickness of the graphene in these areas.
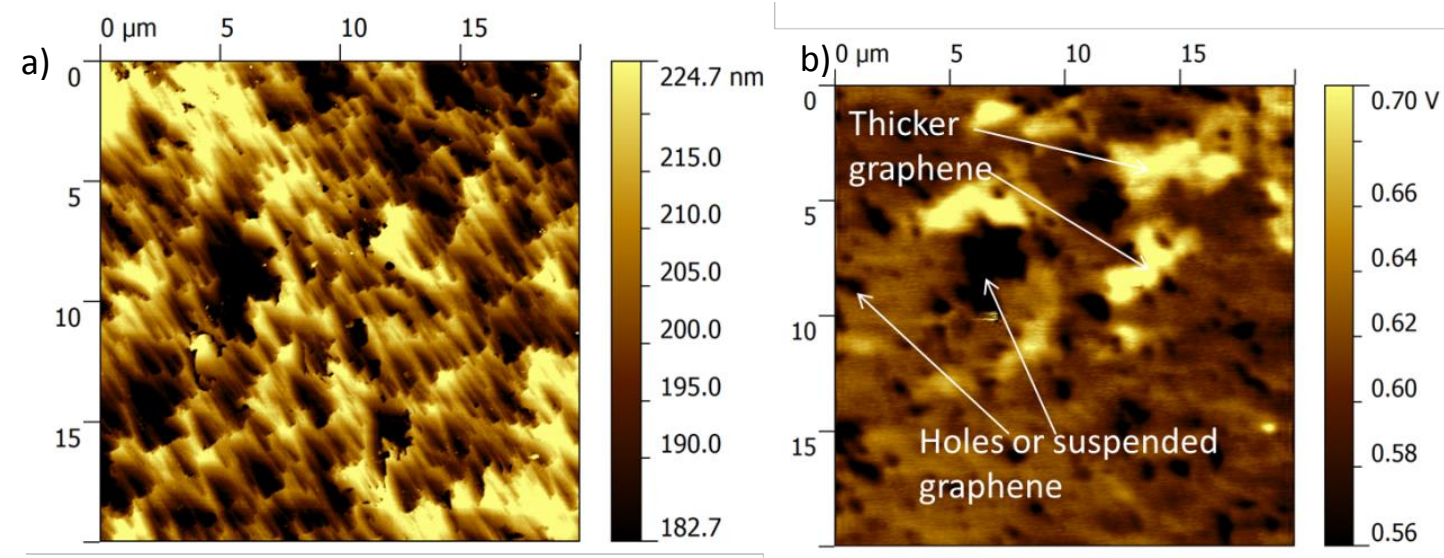

Figure 4: a) topography image of graphene on diamond (111) b) SP image of the same area. 


\section{Conclusions}

We have presented a study of the thickness homogeneity of commercially available CVD graphene films. Graphene produced in this way is demonstrated to be produced as a high quality, nominally mono-layer film. SKPM is a powerful tool for understanding the homogeneity of the electronic properties of the films and the varying layer thickness. The combination of this technique with Raman spectroscopy aids in the interpretation of the results and confirms the predominantly single-layer nature of the graphene. Raman spectroscopy also provides information regarding the stacking of the bi-layer areas. As Raman spectra gives a value averaged over the area of the spot size of the laser it is often challenging to fully understand the spectra without the additional nano-scale information supplied by SKPM. By combining Raman with AFM and SKPM an understanding of the homogeneity of the sample is acquired along with the effects of topography and surface contamination on the electronic properties. Investigating graphene on more exotic substrates requires an understanding of the substrate morphology and how this affects the electronic properties of the graphene atop it. We have demonstrated the homogeneity of a GS grown graphene film showing only small areas with bi-layer formation. We have also demonstrated that the roughness of the diamond substrate affects the uniformity of the electronic properties of the graphene layer.

\section{Acknowledgements}

The authors would like to acknowledge EMRP GraphOhm and MetNEMS projects, EU FET Graphene flagship, the UK NMS and China Scholarship Council (CSC) for funding. The EMRP is jointly funded by the EMRP participating countries within EURAMET and the European Union.

\section{References}

[1] C. Soldano, A. Mahmood, E. Dujardin 2010 Production, properties and potential of graphene Carbon 48 2127-2150

[2] C. Mattevi, H. Kim and M. Chhowalla 2011 A review of chemical vapour deposition of graphene on copper J. Mater. Chem. 21 3324-3334

[3] X. Li et al. 2010 Graphene Films with Large Domain Size by a Two-Step Chemical Vapor Deposition Process Nano Lett. 10 4328-4334

[4] V. Panchal et al 2013 Standardization of Surface Potential Measurements of Graphene Domains Sci.Rep. 32597

[5] J. Eriksson et al. 2012 The Influence of Substrate Morphology on Thickness Uniformity and Unintentional Doping of Epitaxial Graphene on SiC Appl. Phys. Lett. 100 241607-241605.

[6] R. Pearce et al. 2013 On the Differing Sensitivity to Chemical Gating of Single and Double Layer Epitaxial Graphene Explored Using Scanning Kelvin Probe Microscopy ACS Nano 7 4647-4656

[7] A. C. Ferrari \& D.M. Basko 2013 Raman Spectroscopy as a Versatile Tool for Studying the Properties of Graphene Nature Nanotechnology 8 235-246

[8] M.S. Dresselhaus, A. Jorio, and R. Saito 2010 Characterizing Graphene, Graphite, and Carbon Nanotubes by Raman Spectroscopy Annu. Rev. Condens. Matter Phys. 1 89-108

[9] T. M. G. Mohiuddin et al. 2009 Uniaxial strain in graphene by Raman spectroscopy: G peak splitting, Grüneisen parameters, and sample orientation Phys. Rev. B 79205433

[10] A. C. Ferrari and J. Robertson 2001 Resonant Raman spectroscopy of disordered, amorphous, and diamondlike carbon Phys. Rev. B 64075414

[11] A. C. Ferrari et al. 2006 Raman Spectrum of Graphene and Graphene Layers Phys. Rev. Lett. 97, 187401

[12] A. C. Ferrari 2007 Raman spectroscopy of graphene and graphite: Disorder, electron-phonon coupling, doping and nonadiabatic effects Solid State Comm. 143 47-57 
[13] X. Li et al. 2009 Large-Area Synthesis of High-Quality and Uniform Graphene Films on Copper Foils Science. 324, 1312-4

[14]K. J. Al-Fadhalah 2011 Texture and Grain Boundary Character Distribution in a Thermomechanically Processed OFHC Copper J. Eng. Mater. Technol. 134011001

[15] B. Baral, S. S. M. Chan and R. B. Jackman 1996 Cleaning thin-film diamond surfaces for device fabrication: An Auger electron spectroscopic study Vac. Sci.Technol. 142303

[16] O. A. Williams and R. B. Jackman 2004 Homoepitaxial diamond growth for the control of surface conductive carrier transport properties J. Appl. Phys 963742

[17] F. Zhao et. al. 2013 Electronic properties of graphene-single crystal diamond heterostructures $J$. Appl.Phys. 114053709 